\title{
Neurochemical effects of the monoamine oxidase inhibitor phenelzine on brain GABA and alanine: A comparison with vigabatrin
}

\author{
Kathryn G. Todd and Glen B. Baker \\ Neurochemical Research Unit, Department of Psychiatry, University of Alberta, Edmonton, Canada. \\ Received, April 3, 2008; Accepted, May 5, 2008; Published, May 6, 2008.
}

\begin{abstract}
PURPOSE. To compare phenelzine (PLZ), an antidepressant drug with anxiolytic properties which inhibits monoamine oxidase (MAO) but also elevates rat brain levels of the amino acids $\gamma$ aminobutyric acid (GABA) and alanine (ALA), with vigabatrin (VIG), an anticonvulsant which elevates brain GABA by inhibition of GABA transaminase (GABA-T), with regard to their actions on brain levels of GABA and ALA and on activities of MAO, GABA-T and ALA transaminase (ALA-T).
\end{abstract} METHODS. Male rats were administered PLZ (10 $\mathrm{mg} / \mathrm{kg})$ or VIG $(1,000 \mathrm{mg} / \mathrm{kg})$ i.p., and the rats were euthanized 4 hours later and the brains removed for analysis of levels of GABA and ALA (by electron capture gas chromatography after derivatization) and activities of MAO, GABA-T and ALA-T (radiochemical assays). RESULTS. Both PLZ and VIG inhibited GABA-T and elevated GABA levels. Only PLZ inhibited MAO and ALA-T and elevated ALA levels. The effects of PLZ on both amino acids and their transaminases were blocked by pretreatment with the MAO inhibitor tranylcypromine. This pretreament had no effect on the inhibition of GABA-T or the elevation of brain GABA levels produced by VIG. CONCLUSIONS. At the doses studied, PLZ was as effective as VIG at elevating brain GABA levels, but, unlike VIG, also inhibited MAO and ALA-T (and increased brain ALA levels). Pretreatment of rats with the MAO inhibitor tranylcypromine prevented the increase in brain GABA and ALA levels with PLZ, but did not block the effect of VIG on GABA. These observations with tranylcypromine and PLZ support the hypothesis that an active metabolite of PLZ produced by the actions of MAO on this drug plays a major role in its GABAand ALA-elevating actions.

\section{INTRODUCTION}

Phenelzine ( $\beta$-phenylethylhydrazine, PLZ) is a monoamine oxidase (MAO)-inhibiting antidepressant drug that is also effective in treating panic disorder and social anxiety disorder $(1,2)$. PLZ has also been shown to elevate brain levels of the amino acids $\gamma$ aminobutyric acid (GABA) and alanine (ALA) and to inhibit activity of the transaminase enzymes that normally metabolize these amino acids (3-8). It has also been shown to be neuroprotective in an animal model of cerebral ischemia (9). While its ability to elevate brain GABA levels, thereby counteracting the excitotoxic effects of excess released glutamate may account at least in part for its neuroprotective effects in this ischemia model (10), it is also known to prevent neurotoxicity produced by toxic aldehydes in a retinal cell line by sequestering these aldehydes (9). PLZ not only inhibits MAO, but also serves as a substrate for this enzyme (3,11-15) and experiments to date indicate that a metabolite formed by the action of MAO on PLZ plays an important role in the GABA-elevating actions of PLZ $(3,6)$. Although this metabolite has not yet been unequivocally identified, phenylethylidenehydrazine (PEH) is a potential candidate (16). To further elucidate the interactions of PLZ with GABA, in the present study PLZ was compared to vigabatrin (VIG), an anticonvulsant which is a known inhibitor of GABA-T $(17,18)$ that also elevates brain levels of GABA (18-20). Additionally, both VIG and PLZ have been reported to have anxiolytic and neuroprotective properties (9,21-25). In the present report, the two drugs were compared for their effects on GABA and GABA-T at a post-injection time of $4 \mathrm{~h}$, a time interval at which both drugs have been reported to produce marked elevations of brain GABA in rodents $(4,19,20)$.

Corresponding Author: Kathryn G. Todd, Department of Psychiatry (NRU), University of Alberta, Edmonton, Alberta, Canada.E-mail: kgtodd@ualberta.ca 
Concurrently, levels of brain alanine (ALA) and activities of the catabolic enzyme ALA-transaminase (ALA-T) were also measured, as a previous report in the literature have shown that PLZ also inhibits ALA$T$ and increases brain ALA levels $(7,8)$. The effects of pre-administration of the MAO inhibitor tranylcypromine (TCP) on these same parameters were also investigated; this study was of interest as it has been reported that pre-treatment with TCP blocks the GABA- elevating effect of PLZ $(3,6)$, presumably by inhibiting the formation of the active metabolite(s) produced by the actions of MAO on PLZ.

\section{MATERIALS AND METHODS}

\section{Drug Administration:}

All studies were run in accordance with the guidelines of the Canadian Council on Animal Care through the approval of the University of Alberta's Health Sciences Animal Welfare Committee. Male Sprague Dawley rats weighing 250-300 $\mathrm{g}$ with free access to food and water were randomly assigned $(n=6)$ to one of the following groups: VIG (1000 mg/kg); PLZ (10 $\mathrm{mg} / \mathrm{kg}$ ) or distilled water vehicle (VEH). These drug doses were chosen based on preliminary experiments in our laboratories that showed at the time intervals used in the current studies, a $1000 \mathrm{mg} / \mathrm{kg}$ dose of VIG was required to give approximately the same GABA elevation as $10 \mathrm{mg} / \mathrm{kg}$ of PLZ. Animals were administered VIG, PLZ, or VEH via intraperitoneal (ip) injection and sacrificed by decapitation $4 \mathrm{~h}$ later. The brains were then rapidly removed, flash frozen in ice-cold isopentane on solid carbon dioxide and stored at $-80^{\circ} \mathrm{C}$ until analysis.

In a separate study, the effects of pretreatment with TCP were evaluated by ip administration of either TCP $(1 \mathrm{mg} / \mathrm{kg})$ or VEH $1 \mathrm{~h}$ prior to injection of VIG, PLZ or VEH depending on random group assignment. Administration of this dose of TCP is well known to inhibit monoamine oxidase markedly within one hour (e.g. reference 6). Four hours after the second injection (VIG, PLZ or $\mathrm{VEH}$ ), the animals were sacrificed by decapitation and the brains processed as described below. All doses are expressed as free base weight. At the time of analysis, the frozen brains were hemisected and one-half homogenized in 5 volumes of distilled water and divided into portions for analysis of activities of MAO-A and -B and GABA-T or ALA-T using the radiochemical methodologies of Wurtman and Axelrod (26) for MAO and Sterri and Fonnum (27) for the amino acids. The remaining half brains were homogenized in ice-cold $0.1 \mathrm{~N}$ perchloric acid containing $10 \mathrm{mg} \%$ EDTA and $0.05 \mathrm{mM}$ ascorbic acid. This homogenate was then centrifuged at 12,000 $\mathrm{x} \mathrm{g}$ for $15 \mathrm{~min}$ at $4^{\circ} \mathrm{C}$ to remove the protein precipitate. Portions of the supernatant were then used for simultaneous analysis of GABA and ALA levels employing the electron-capture gas chromatographic methodology of Wong et al (28) which utilizes derivatization with a fluorinated reagent prior to analysis.

Statistical analysis consisted of applying oneway and two-way ANOVA (for the drug combinations) to the treatment means of all groups. Post hoc Newman-Keuls comparisons were applied to data showing significant treatment main effects and interactions. A $p$ value of $<0.05$ was used to determine statistical significance.

\section{RESULTS}

Results from the MAO-A and -B assays demonstrated that VIG did not inhibit either of these isozymes. On the other hand, animals treated with PLZ showed, as expected, a marked inhibition of both MAO-A and -B (93 and 85\% inhibition respectively).

Treatment with either VIG or PLZ resulted in more than a doubling of GABA levels (Figure 1a). There was no significant difference between the levels of GABA from the VIG-treated animals as compared to the PLZ-treated animals ( $p>0.05$ ). Brain GABA-T activity was significantly inhibited by both VIG (42\% inhibition) and PLZ (32\% inhibition) (Figure 1B). Animals treated with PLZ showed an increase in brain ALA levels to approximately $250 \%$ of control values whereas ALA levels obtained from animals treated with VIG were similar to those obtained from control animals (Figure 1A). Corresponding to these data, PLZ treatment significantly inhibited ALA-T activity (28\% inhibition), while VIG treatment did not (Figure 1B).

In the TCP-pre-treatment study, GABA levels obtained from the control group $\mathrm{VEH} / \mathrm{VEH}$, and the TCP/VEH- and TCP/PLZ- treated groups were not significantly different from each other, with values ranging from 230 to $260 \mu \mathrm{g} / \mathrm{g}$ tissue (Figure 2A). On the other hand, animals treated with the combinations of VEH/VIG, VEH/PLZ and TCP/VIG showed significantly elevated GABA levels to almost 3 times control levels (values ranging from 612 to $660 \mu \mathrm{g}$, $\mathrm{p}<0.05)$. These data indicate that TCP completely 
blocked the GABA-elevating action of PLZ, but had no effect on the GABA-elevating action of VIG.

The data obtained from analyses of GABA-T activity (Figure 2B) parallel those described above in that only the groups treated with the combinations of VEH/VIG, VEH/PLZ or TCP/VIG showed significant inhibition of GABA-T activity. There were no significant differences between the TCP/VEH and TCP/PLZ-treated animals compared to the VEH/VEH control group $(p<0.05)$. In other words, pre-treatment with TCP blocked the GABA-T-inhibiting actions of
PLZ but had no effect on the GABA-T-inhibiting properties of VIG.

Results obtained after analyses of brain ALA levels and ALA-T activity in animals treated with the drug combinations are displayed in Figure 3. Only the VEH/PLZ group had significantly elevated ALA levels compared to the VEH/VEH control group $(p<0.05)$. Similarly, only the VEH/PLZ-treated group showed a significant decrease in ALA-T activity as compared to the VEH/VEH control group $(\mathrm{p}<0.05)$.

\section{Todd and Baker Figure 1.}
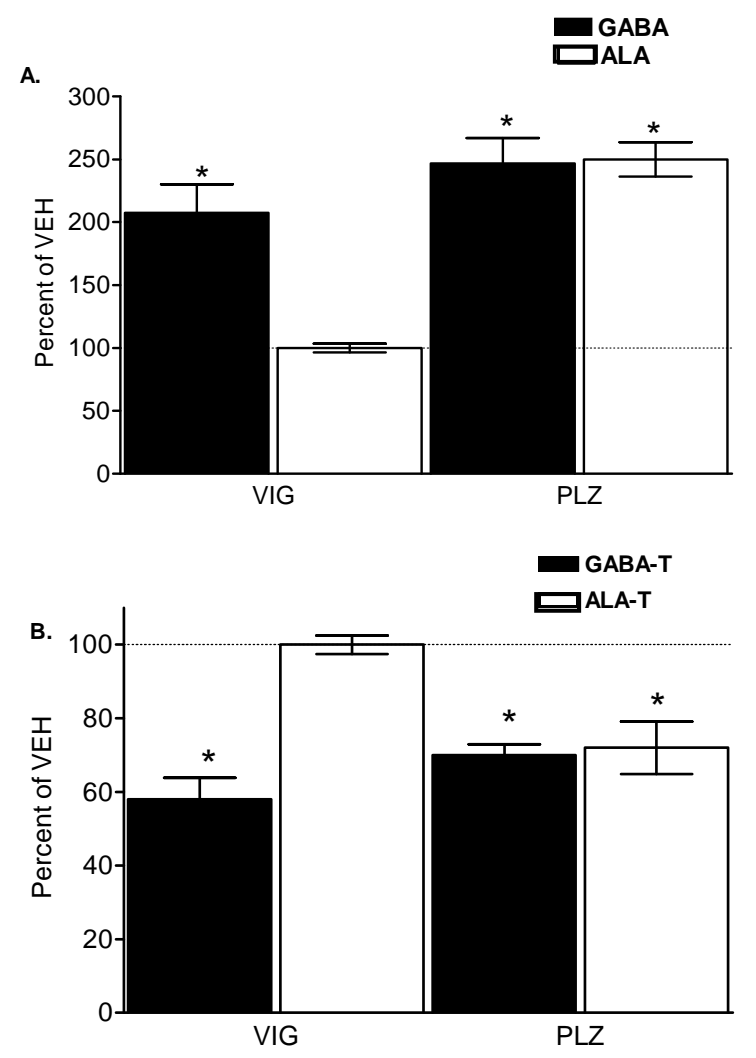

Figure 1. A) Brain GABA and ALA levels from rats treated with VIG $(1000 \mathrm{mg} / \mathrm{kg})$ or PLZ $(10 \mathrm{mg} / \mathrm{kg}) 4 \mathrm{~h}$ previously. Histograms represent $\%$ of VEH \pm SEM ( $\mathrm{n}=6$ ). (Mean control levels were $295 \pm 28 \mu \mathrm{g} / \mathrm{g}$ tissue and $55 \pm 4 \mu \mathrm{g} / \mathrm{g}$ for GABA and ALA, respectively). B) Inhibition of brain GABA-T and ALA-T from rats treated with VIG $(1000 \mathrm{mg} / \mathrm{kg})$ or PLZ (10 $\mathrm{mg} / \mathrm{kg}) 4 \mathrm{~h}$ previously. Histograms represent $\%$ of VEH $\pm \mathrm{SEM}(\mathrm{n}=6) . *$ Denotes significantly different from VEH. 


\section{Todd and Baker Figure 2}
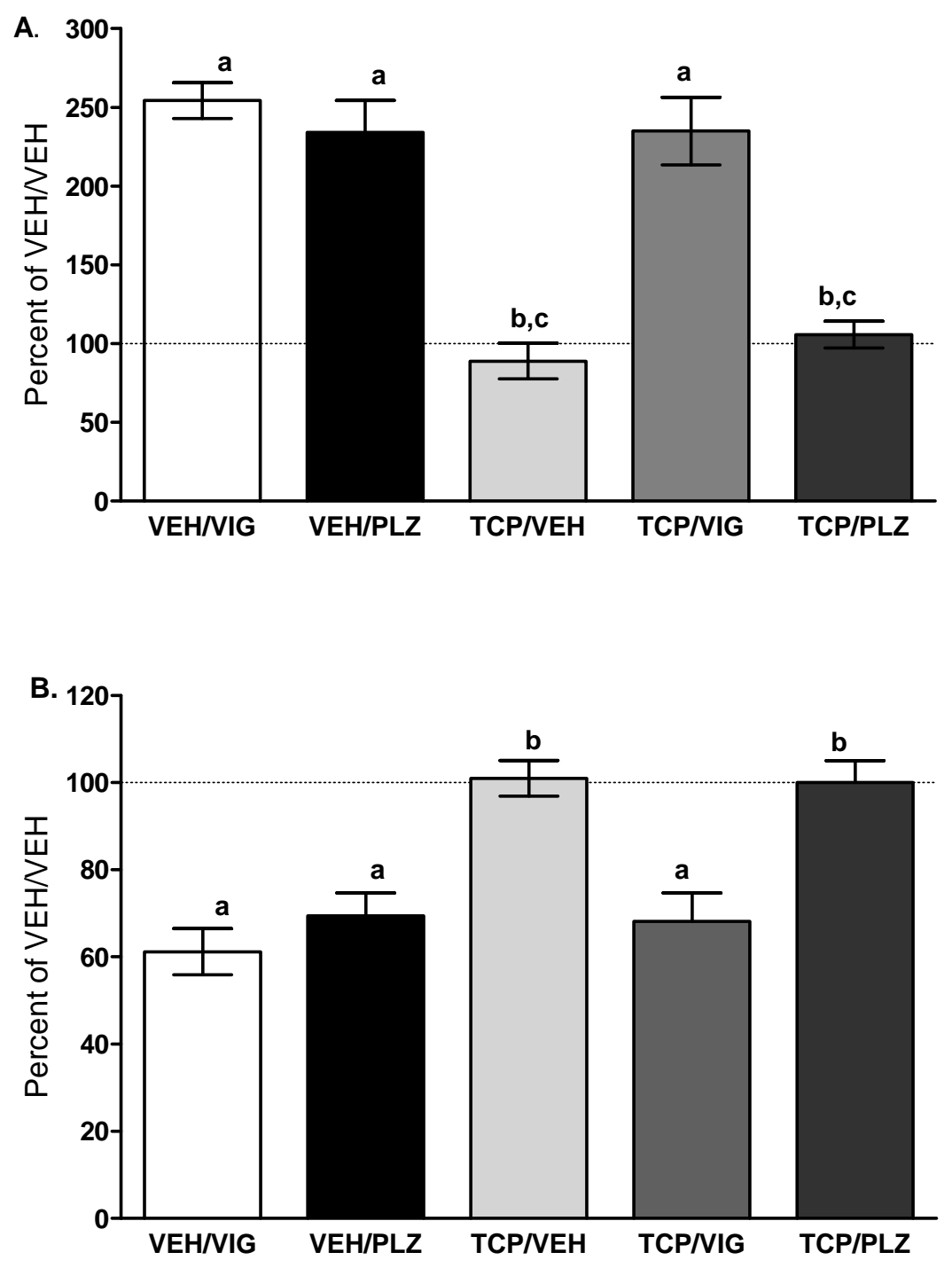

Figure 2. A) Brain GABA levels in rats treated with drug combinations. Rats were pre-treated with TCP or VEH $1 \mathrm{~h}$ before treatment with VIG or PLZ, and were killed 4 h later. Doses: VIG $1000 \mathrm{mg} / \mathrm{kg}$; PLZ $10 \mathrm{mg} / \mathrm{kg}$; TCP $1 \mathrm{mg} / \mathrm{kg}$. Histograms represent $\%$ of VEH/VEH \pm SEM ( $n=6$ ). a, significantly different from VEH/VEH; $b$, significantly different from VEH/VIG; c, significantly different from VEH/PLZ (VEH/VEH control values were $231 \pm 20 \mu \mathrm{g} / \mathrm{g}$ tissue).B) Brain GABA-T inhibition in rats treated with drug combinations (VIG $1000 \mathrm{mg} / \mathrm{kg}$; PLZ $10 \mathrm{mg} / \mathrm{kg}$; TCP $1 \mathrm{mg} / \mathrm{kg}$, n=6). Histograms represent \% of VEH/VEH activity ( \pm SEM). a, significantly different from VEH/VEH; b, significantly different from VEH/PLZ. 


\section{Todd and Baker Figure 3}

A.
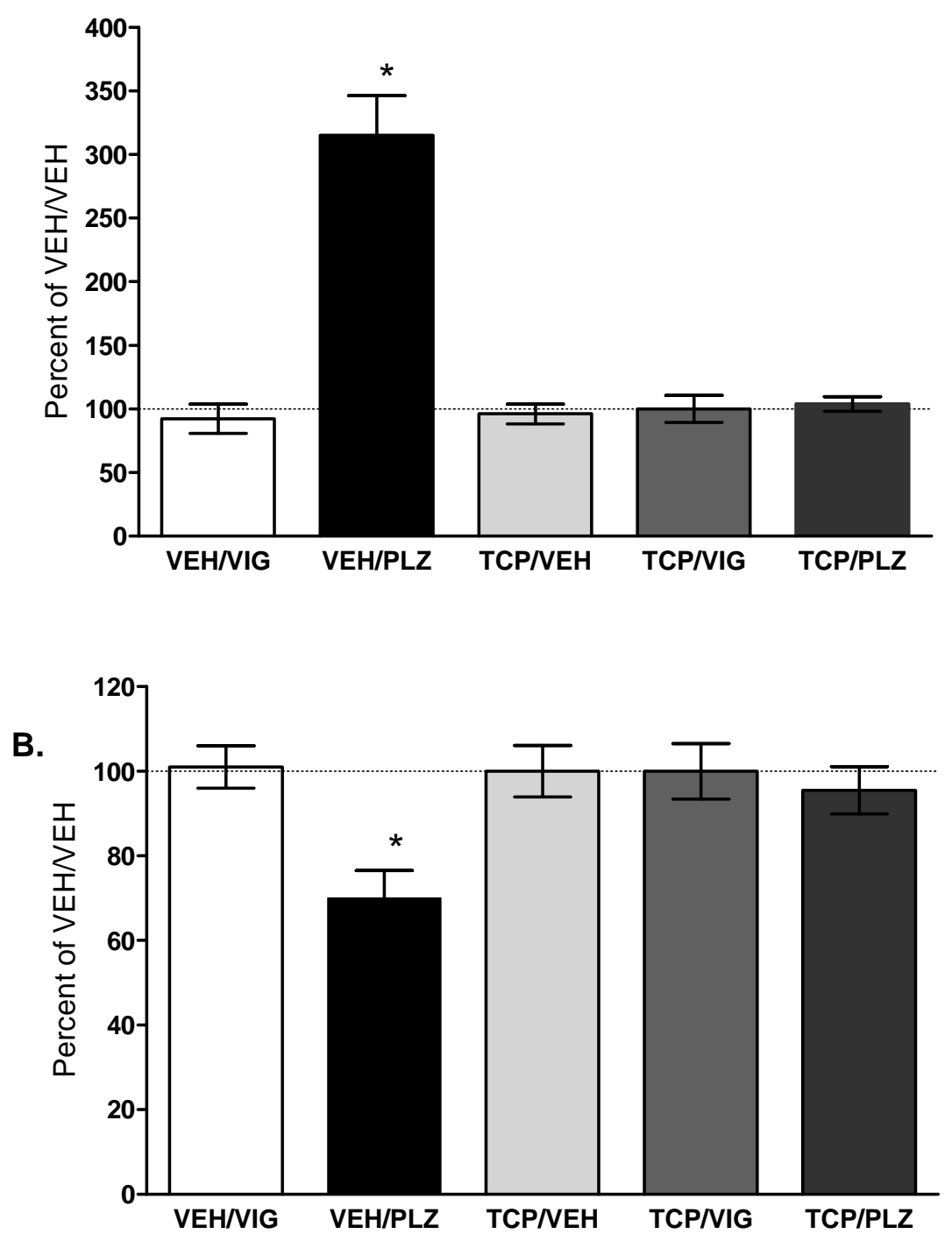

Figure 3. A) Brain ALA levels in rats treated with drug combinations. Rats were pre-treated with TCP or VEH $1 \mathrm{~h}$ before treatment with VIG or PLZ, and were killed 4 h later. Doses: VIG $1000 \mathrm{mg} / \mathrm{kg}$; PLZ $10 \mathrm{mg} / \mathrm{kg}$; TCP $1 \mathrm{mg} / \mathrm{kg}$. Histograms represent $\%$ of VEH/VEH \pm SEM ( $\mathrm{n}=6$ ). (VEH/VEH control values were $55 \pm 3 \mu \mathrm{g} / \mathrm{g}$ tissue).B) Brain ALA-T inhibition from rats treated with drug combinations. Rats were pre-treated with TCP or VEH $1 \mathrm{~h}$ before treatment with VIG or PLZ, and were killed $4 \mathrm{~h}$ later. Doses: VIG $1000 \mathrm{mg} / \mathrm{kg}$; PLZ $10 \mathrm{mg} / \mathrm{kg}$; TCP $1 \mathrm{mg} / \mathrm{kg}$. Histograms represent $\%$ of VEH $/ \mathrm{VEH}$ activity $\pm \mathrm{SEM}$ $(\mathrm{n}=6) .{ }^{*}$ Denotes significantly different from VEH/VEH. 


\section{DISCUSSION}

The results from this investigation comparing the ex vivo effects of VIG and PLZ confirmed that VIG has no effect on MAO while PLZ potently inhibited both MAO-A and MAO-B. VIG appears to be relatively selective for GABA-T in that it has been reported to not inhibit other transaminases such as aspartate transaminase and liver ornithine transaminase (29). However, it has been reported that VIG decreases brain levels of glutamate and aspartate at doses that induce an elevation of brain GABA, although the exact mechanisms involved are not known (19). The present investigation showed that VIG, in contrast to PLZ, had no effect on the activity of ALA-T or on levels of ALA in rat brain.

Our results also showed that treatment with VIG or PLZ at the doses tested resulted in similar elevations of brain GABA levels, and a similar degree of inhibition of GABA-T. Interestingly, these increases in GABA levels occurred when GABA-T was inhibited by only $30-40 \%$. A similar finding was reported by an earlier study by Popov and Matthies (3). It has been demonstrated that PLZ, at doses that result in elevated brain GABA levels in the rat, shows anxiolytic effects on the elevated plus maze test (22) and offers significant neuroprotection in an animal model of global ischemia (9). Vigabatrin has also been reported to have anxiolytic and neuroprotective actions $(9,24,25)$. To our knowledge, it has not yet been demonstrated that PLZ, or the proposed metabolite PEH, has, like VIG, anti-convulsant properties; such knowledge would have important clinical relevance as several antidepressants actually increase the susceptibility of patients to convulsions. Vigabatrin is used in treatment of infantile spasm and as adjuctive therapy in adults with partial epilepsy which has been refractory to treatment with other anticonvulsants (30,31). Vigabatrin has also been reported to produce visual field defects and other visual disturbances (30 and 31 and references therein), but to our knowledge similar visual problems have not been reported with PLZ despite its clinical use for many years.

Results from the present study showed that pre-treatment with TCP blocked the GABA-elevating effects of PLZ, but not of VIG. Earlier studies found a similar pattern when comparing the effects of pretreatment of TCP on the GABA-elevating actions of PLZ and aminooxyacetic acid, a GABA-T inhibitor
(3) that is, TCP reversed the action of PLZ, but had no effect on aminooxyacetic acid. These observations further support the supposition that a metabolite of PLZ, produced by the action of MAO on PLZ $(3,6)$, contributes to the GABA-elevating action of this drug. The results of the present investigations indicate that this metabolite also contributes to the inhibition of ALA-T activity and increased brain ALA levels observed following administration of PLZ. Currently, there is a paucity of knowledge regarding the role of ALA in neurotransmission or its possible involvement in the aetiology of depression or panic disorder. However, ALA is related metabolically to lactate, which has been shown to produce panic attacks in patients with panic disorder (32). Moreover, it has been reported that lactate infusion in human subjects results in increased brain lactate levels (33). Thus, it is possible that the antipanic effects of PLZ may be due, at least in part, to a reduction in the formation of lactate from pyruvate via the elevation of ALA.

\section{CONCLUSION}

Taken together, the results from the present experiments confirm that the GABA-elevating effects of PLZ in rat brain are similar to those of VIG and are due, at least in part, to the inhibition of GABA-T. It is also of interest that this effect occurs at a much lower dose than is required for VIG. While PLZ is also an inhibitor of ALA-T and increases rat brain ALA levels, VIG has no such effect at the dose tested. Elevation of brain levels of both GABA and ALA by PLZ can be blocked by the prior treatment with the MAO inhibitor TCP, providing further support that a metabolite of PLZ, produced by the action of MAO on the parent drug, plays a major role in the GABA(and ALA-) elevating action of PLZ.

\section{ACKNOWLEDGMENTS}

Funding was provided by the Canadian Institutes of Health Research, the George and Dorothy Davey Endowment Fund and the Canada Research Chairs program. The authors are grateful to Ms. Gail Rauw for technical support and to Tara Checknita for secretarial assistance. Vigabatrin was kindly provided by Marion Merrell-Dow (now Aventis Pharma) 


\section{REFERENCES}

[1]. Johnson MR, Lydiard RB and Ballenger JC MAOIs in panic disorder and agoraphobia. In Kennedy SH (Ed.), Clinical Advances in Monoamine Oxidase Inhibitor Therapies, American Psychiatric Press Inc., Washington, DC; pp. 205224, 1994.

[2]. Gitow F, Lietowitz MR and Schneier FR. MAOI therapy of social phobia. In: Kennedy, S.H. (ed.). Clinical Advances in Monoamine Oxidase Inhibitor Therapies, American Psychiatric Press, Inc., Washington, D.C., pp. 225-254, 1994.

[3]. Popov $\mathrm{N}$ and Matthies $\mathrm{H}$, Some effects of monoamine oxidase inhibitors on the metabolism of $\gamma$-aminobutyric acid in rat brain. J Neurochem, 16:899-907, 1969.

[4]. Baker GB, Wong JT-F, Yeung JM and Coutts RT, Effects of the antidepressant phenelzine on brain levels of $\gamma$-aminobutyric acid (GABA). J Affect Dis 21:207-211, 1991.

[5]. McManus DJ, Baker GB, Martin IL, Greenshaw AJ and McKenna KF, Effects of the antidepressant/antipanic drug phenelzine on GABA concentrations and GABA-transaminase activity in rat brain. Biochem Pharmacol, 43:2486-2489, 1992.

[6]. Todd KG and Baker GB. GABA-elevating effects of the antipanic/antidepressant drug phenelzine: effects of pre-treatment with tranylcypromine, (-)deprenyl and clorgyline. J Affec Disord, 35:125129, 1995

[7]. Wong JTF, Baker GB, Coutts RT and Dewhurst WG. Long-lasting elevation of alanine in brain produced by the antidepressant phenelzine. Brain Res Bull, 25:179-181, 1990.

[8]. Tanay VA-MI, Parent MB, Wong JTF, Paslawski T, Martin IL and Baker GB. Effects of the antidepressant/antipanic drug phenelzine on alanine and alanine transaminase in rat brain. Cell Mol. Neurobiol. 2001; 21: 325-339.

[9]. Wood P, Khan MA, Moskal JR, Todd V, Tanay AMI and Baker GB. Aldehyde load in ischemiareperfusion brain injury: Neuroprotection by neutralization of reactive aldehydes with phenelzine. Brain Res., 1122, 184-190, 2006.

[10]. Sowa B, Todd KG, Tanay VAM-I, Holt A and Baker GB Monoamine oxidase inhibitors and development of neuroprotective drugs. Current Neuropharmcol., 2: 153-168, 2004.

[11]. Clineschmidt BV and Horita A, The monoamine oxidase catalyzed degradation of phenelzine-1-14C, an irrerversible inhibitor of monoamine oxidase -- I. Studies in vitro. Biochem Pharmacol, 18:1011-1020, 1969
[12]. Clineschmidt BV and Horita A, The monoamine oxidase catalyzed degradation of phenelzine-1-14C, an irrerversible inhibitor of monoamine oxidase -II. Studies in vivo. Biochem Pharmacol, 18:1021-1029, 1969.

[13]. Tipton KF and Spires IPC, Oxidation of 2phenylethylhydrazine by monoamine oxidase. Biochem Pharmacol., 21:268-270, 1972.

[14]. Patek DR and Hellerman L, Mitochondrial monoamine oxidase. Mechanism of inhibition by phenylhydrazine and by aralkylhydrazines. Role of enzymatic oxidation. J Biol Chem.,, 249:23732380, 1974.

[15]. Strolin Benedetti M and Dostert P, Contribution of amine oxidases to the metabolism of xenobiotics. Drug Metab Rev., 26:507-535, 1994.

[16]. Paslawski T, Knaus E, Iqbal N, Coutts RT and Baker GB. $\beta$-Phenylethylidenehydrazine, a novel inhibitor of GABA transaminase. Drug Devel. Res, 54:35-39, 2001.

[17]. Hammond EJ and Wilder BJ Minireview, Gamma-vinyl GABA. Gen Pharmacol, 16:441-447, 1985.

[18]. Perry TL, Kish SJ, Hansen S, Wright JM, Wall RA, Dunn WL and Bellward GD, Elevation of brain GABA content by chronic low-dosage administration of hydrazine, a metabolite of isoniazid. J Neurochem, 37:32-39, 1981.

[19]. Bernasconi R, Klein M, Martin P, Christen P, Hafner T, Portet $\mathrm{C}$ and Schmutz M, $\gamma$-Vinyl GABA: comparison of neurochemical and anticonvulsant effects in mice. J Neural Transm, 72:213-233, 1988.

[20]. Schecter PJ, Tranier Y and Grove Y, Attempts to correlate alteration in brain GABA metabolism by GABA-T inhibitors with their anticonvulsant effects. Adv Exp Med Biol, 123:43-57, 1979.

[21]. Sayin Ü, Purali N, Özkan T, Altug T and Büyükdevrim S, Vigabatrin has an anxiolytic effect in the elevated plus-maze test of anxiety. Pharmacol. Biochem. Behav., 43: 529-535, 1992.

[22]. Paslawski T, Treit D, Baker GB, George M and Coutts RT, The antidepressant drug phenelzine produces antianxiety effects in the plus-maze and increases in rat brain GABA. Psychopharmacology, 127:19-24, 1996

[23]. Griebel G, Curet O, Perrault G and Sanger DJ, Behavioral effects of phenelzine in an experimental model for screening anxiolytic and anti-panic drugs; correlation with changes in monoamine-oxidase activity and monoamine levels. Neuropharmacology, 37: 927-935, 1998.

[24]. Shuaib A, Ljaz S, Hasan S and Kalra J. Gama-vinyl GABA prevents hippocampal and substantia nigra reticulata damage in repetitive transient forebrain ischemia. Brain Res. 590:13-17, 1992. 
[25]. Zwanzger $\mathrm{P}$ and Rupprecht R. Selective GABAergic treatment for panic? Investigations in experimental panic induction and panic disorder. $\mathrm{J}$ Psychiatry Neurosci., 30(3): 167-175, 2005.

[26]. Wurtman RJ and Axelrod JA sensitive and specific assay for the estimation of monoamine oxidase. Biochem Pharmacol, 12:1439-1441, 1963.

[27]. Sterri SH and Fonnum F, Isolation of organic anions by extraction with liquid anion exchangers and its application to micromethods for acetylcholinesterase and 4-aminobutyrate aminotransferase. Eur J Biochem, 91:215-222, 1978.

[28]. Wong JTF, Baker GB and Coutts RT, A rapid, sensitive assay for $\gamma$-aminobutyric acid in brain using electron-capture gas chromatography. Res Commun Chem Path Pharmacol, 70:115-124, 1990.

[29]. Jung MJ, Lippert B, Metcalf BW, Bohlen P and Schecter PJ, Gamma-vinyl GABA (4-aminohex-5-enoic acid), a new selective irreversible inhibitor of GABA-T: Effects on brain GABA metabolism in mice. $J$ Neurochem, 29:787-802, 1977.

[30]. Wild JM, Ahn HS, Baulac H, Bursztyn J, Chiron C, Gandolfo E, Safran A, Schiefer U, Peruccs E. Vigabatrin and epilepsy: lessons Learned. Epilepsia, 48(7):1318-1327, 2007.

[31]. Paul SR, Krauss GL, Miller NR, Medura M, Miller TA and Johnson MA. Visual function is stable in patients who continue long-term vigabatrin therapy: implications for clinical decision making. Epilepsia, 42(4): 525-530, 2001

[32]. Coupland NJ and Nutt DJ, Neurobiology of anxiety and panic. In: Bradwejn J and Vasar E (Eds.), Cholecystokinin and Anxiety: From Neuron to Behavior. Springer, New York, NY. pp1-32, 1995.

[33]. Dagen SR, Marro KI, Richards TL, Metzger GD, Preliminary application of magnetic resonance spectroscopy to investigate lactate-induced panic. Am. J. Psychiat, 51:57-63, 1994. 